\title{
Author's reply: Trends in Human Leptospirosis in Denmark, 1980 to 2012
}

L B van Alphen ${ }^{1,2}$, S Ethelberg³, S Villumsen 4 , K A Krogfelt (KAK@ssi.dk)1

1. Department of Microbiology and Infection Control, Statens Serum Institut, Copenhagen, Denmark

2. Current address: Department of Medical Microbiology, Maastricht University Medical Center, Maastricht, the Netherlands

3. Department of Infectious Diseases Epidemiology, Statens Serum Institut, Copenhagen, Denmark

4. Department of Infectious Diseases, University Hospital Rigshospital, Copenhagen, Denmark

van Alphen LB, Ethelberg S, Villumsen S, Krogfelt KA. Author's reply: Trends in Human Leptospirosis in Denmark, 1980 to 2012. Euro Surveill. 2015;20(9):pii=21054. Available online: http://www.eurosurveillance.org/ViewArticle.aspx?Articleld=21054

\section{To the editor:}

We would like to thank Drs Goris and Hartskeerl for their interest in our paper. In Denmark, Patoc has traditionally been used as a functional classification group, exhibiting cross-reactivity to a large number of Leptospira serogroups. Therefore, while we are aware of the nomenclature of Leptospira, we chose nevertheless to use the term Patoc for this overview of more than 30 years of leptospirosis cases. It would have been relevant to underline in the abstract that reactivity with a titre $\geq 100$ towards serovar Patoc should be seen as a "probable infecting serogroup that could not be determined'.

Indeed, leptospirosis is a neglected disease in Europe and deserves attention. We agree that persistent complaints after acute leptospirosis must not be ignored. Yet, the true burden of both acute and chronic leptospirosis and the pathogenesis of persistent complains is not yet clear and chronicity has largely been described through individual case studies. The disease needs more attention, in order to improve our knowledge on this issue. While it is well established that the causative organism Leptospira spp. is endemic in the animal reservoirs in Europe, the number of diagnosed human cases is very low. This can partly be explained by the unspecific symptoms of the disease and the fact that the diagnosis may often not be considered as shown for leptospirosis among travellers returning from the tropics [1].

An example of a probably cause of climate changeassociated cases also in Denmark is an unusually heavy rainstorm that occurred over Copenhagen in July 2011. Sewer overflow led to severe flooding in the city and within buildings, which resulted in an increased number of acute leptospirosis cases. Five persons with leptospirosis were notified; two were admitted to hospital and one patient died [2]. This event underlines the potential for prevention, stated in our paper, that can be achieved by raising awareness about the infection both among doctors and those at risk of infection.

\section{Conflict of interest}

None declared.

Authors' contributions

All authors were equally involved in writing this letter.

References

1. Askling HH, Lesko B, Vene S, Berndtson A, Bläckberg PJ, Bronner U, Follin P, Hellgren U, Palmerus M, Ekdahl K, Tegnell A, Struwe J. (2009). Serologic Analysis of Returned Travelers with Fever, Sweden. Emerg Infect Dis. Nov; 15(11): 1805-1808. doi: $10.3201 /$ eid1511.091157

2. EPI-News . Statens Serum Institut (2012): Theme day: Copenhagen rainstorm 2 July 2011. EPI-News, 2012, Week 5. 